\title{
A Usability Evaluation of Arabic Mobile Applications Designed for Children with Special Needs - Autism
}

\author{
Lyan Al-Wakeel, Amjad Al-Ghanim, Shaden Al-Zeer, and Khalid Al-Nafjan
}

\begin{abstract}
Recent years have witnessed major advances in technologies to support children diagnosed with Autism Spectrum Disorders (ASD). A number of applications have impacted their daily lives, with the goal of enhancing their abilities to understand, interact and communicate with others. The usability of mobile applications developed for people with ASD is important because this group is usually lacking in experience and familiarity with many aspects of new technologies. This paper focuses on comparing the usability of two Arabic mobile applications available in the Apple Store (iPad). Usability of these applications is analyzed using eye tracking and Morae ${ }^{\circledR}$. Measurement tools are used to collect qualitative and quantitative data and determine the participants' satisfaction with products. At the end, we presented proposed recommendation for optimal design based on the result of this study.
\end{abstract}

Index Terms-Autism, eye tracking, iPad, Morae ${ }^{\circledR}$.

\section{INTRODUCTION}

Autism is known as a complex developmental disability. People with autism have issues with verbal communication, a limited range of social interactions, and activities that include an element of play and/or banter [1]. ASD stands for Autism Spectrum Disorder, a set of situations that affect the daily functions of people's lives [2]. They include impairments in social interaction, communication, repeated behavior, interests, and activities [3]. The Autism Society of America defines autism as a "complex developmental disability that typically appears during the first 3 years of life and is the result of a neurological disorder that affects the normal functioning of the brain, impacting development in the areas of social interaction and communication skills"[4].

With increasing numbers of children, being diagnosed with Autism Spectrum Disorders (ASD) [5], a variety of mobile applications have been developed to enhance social skills for children with ASD. Our research has concentrated on studying the usability of two Arabic mobile applications designed to enhance the social skills of children with ASD.

The use of the multi touch tablet, such as the iPad, is increasing at an exponential rate [6]. This is likely due to the cost effectiveness and market penetration of the iPad device

Manuscript received May 18, 2014; revised August 5, 2014. This work was supported in part by the King Saud University. Also, we would like to thank Dr. Areej Al-Wabil and Dr. Khalid for their help and advice. We extend our appreciation to the Deanship of Scientific Research at King Saud University for funding the work through the research group project number RGP-VPP-157. A Usability Evaluation of Arabic Mobile Applications Designed for Children with Special Needs - Autism

The authors are with King Saud University, Saudi Arabia (e-mail: lalwakeel@ut.edu.sa, amjad.alghanim@gmail.com, salzeer@su.edu.sa, kalnafjan@ksu.edu.sa).
[6]. In addition, mobile applications are socially accepted and simple to use and hold for children with ASD. Mobile applications are software applications, usually designed to run on smartphones and tablet devices [7]. Mobile applications are available through distribution platforms, which are typically operated by the owner of the mobile operating system, such as the Apple App Store, Android Market, and BlackBerry App World. Some applications are free, while others require payment. Usually they are downloaded from the platform to a target device such as an iPad, BlackBerry, or Android phone [8].

Today, there are a large number of new mobile applications but many of them are difficult to use. To determine an application's ease of use, usability measurements must be applied. The term "usability" is defined by the International Organization for Standardization (ISO) 9241-11 [9] as "the extent to which a product can be used with effectiveness, efficiency and satisfaction in a specified context of use."

- Effectiveness is the accuracy and completeness with which specified users can achieve their goals in a particular environment.

- Efficiency is the amount of resources expended in relation to the accuracy and completeness of the goal achieved.

- Satisfaction is the comfort and acceptability of the work system to its users and other people affected by its use.

This paper presents the usability evaluation of Arabic applications developed for children with ASD (ages 3-12), their teachers, parents, and non-autistic children. Usability of these applications is analyzed on the basis of using the eye tracking measurement and Morae. The first application is "Touch to Speak," an application developed by undergraduate students in the IT department of King Saud University (KSU). The second application is "Tap to Talk," which supports multiple languages such as Arabic, English, and French. We chose these two applications because they have similar functions but differing designs. The two applications are forms of augmentative and alternative communication (AAC), which comprises all forms of communication that are used to express thoughts, needs, wants, and ideas. ACC includes special augmentative aids, such as pictures and symbol communication boards and electronic devices. These aids are available to help people express themselves [10]. While the two applications are designed for children with ASD, because ACC helps people with severe speech or language problems, other users can also use it to learn Arabic.

The rest of the paper is organized as follows: Section II discussion of related work. Section III description of our research methodology. Section IV comparative analysis 
between two existing apps for autism and the result. Section V, Section VI and Section VII, will cover the discussion, future work and conclusion in order.

\section{RELATED WORK}

As mobile technology evolves, many applications are created to serve people with any type of disabilities. Yamagata et al. (2013) studied the effectiveness of mobile applications to help dementia and Alzheimer patients. Dementia is a disorder in the brain that causes memory loss and lowers the cognitive functioning. Alzheimer's disease (AD) is a type of dementia. The researchers conducted a usability study to analyze the effectiveness of mobile applications developed to increase their cognitive functionalities.

Patients with dementia and AD can use existing applications that have several different visual and verbal AAC methods to improve their ability to communicate with other people. AAC also helps autism people and people who have difficulties with social communication. Using these methods people with dementia and AD can improve their cognitive skills [11], [12].

The researchers selected multiple participants with dementia and $\mathrm{AD}$ to conduct their usability study. They used Jungle app, which shows an animal's image in a jungle setting. When participants touch a selected image, the animal's name appears and the sound is played. The researchers found that people with dementia or AD quickly learned to use the iPad but they had difficulty recognizing the difference between a tablet that used a pen or finger touch, and for some of them, we had to remind them how to use it. But in general using this application increases cognitive function [13].

M. Barry et al. (2008) evaluated the feasibility of using games in the educational field for children with special needs [13]. From their research, F. S. Din et al. (2001) found that "computer-related technology use plays a positive role in young children's social, psychological, cognitive and academic development" [14]. M. Barry et al. in their earlier study were observing the children, interviewing the tutors and examining the software. The researchers observed that autistic children are satisfied with using technology while playing. Then they evaluated an educational game for children with autism. They applied observation techniques, noting that using a survey was not practical for autistic people [13]. Clanton (1998) proposed three techniques to evaluate the usability of games: game interface, game mechanics and game play. Game interface relates to anything that allows player to interact with the games, e.g. the device. Game mechanics relates to any object's representation, and the movement and interaction method to trigger these objects. Game play is the activity with which players reached the goals of game [15]. M. Barry et al. (2008) found some issues with the usability of an unconstrained game interface for autistic people. Given that autistic people prefer a certain routine and predictable environment, the video scenes were disorienting to them. Autistic people also they tend to be visual learners and do not always respond well to audio cues. For the game mechanics and game play, the developer must consider the ease of use for people with autism, as they require a certain level of difficulty [13].

S. Khan et al. (2013) conducted a usability study for two smartphone applications for people with autism, CommApp (an iPhone application) and AAC speech communicator (an Android application). They studied a sample of 50 autistic people, requesting them to use these two applications, and later administered a survey to their parents or guardians [16]. The authors presented the results from the users' perspective. The users gave opinions in 5 criteria: ease of use, learnability, feedback, error message, help and documentation, and appealing interface. They all agreed that CommApp was easier to use and learn than ACC speech communicator because of its friendly interface design. However, the majority disagreed about the rest of the criteria for both applications. Also, the authors asked the users about their opinions of "good," "bad" and "must-have" features. They agreed that CommApp has an appealing interface and easier navigation, but stated that its categories are too limited and it needs to have a clearer admin section and better-labeled buttons and icons [16].

The ACC speech communicator has more categories but users found them ambiguous. Users also found the interface inconvenient and difficult to use and learn. But its sentences are properly formulated, which users see as a good feature [16]. Users also suggested that these two applications would be more useful if they could add custom images and audio about familiar places and people. Additionally, users observed that the developers of ACC speech communicator need to fix the tendency to crash after the user taps on its icon and to provide tutorials to users on how to use this application, because it is difficult to use and learn [16].

In summary, if mobile applications are convenient to use for people with dementia and $\mathrm{AD}$, we think that autistic children might also find them beneficial. According to the second study, autistic people enjoy using technology, so using a mobile application might not cause a problem for them. However, it is important to choose applications with characteristics of autism in mind, taking into consideration that interface design and varied categories will be important for them. Because autistic people may depend on visual representations to recognize familiar places and their families, we should also consider the ability to add custom images to the applications. Finally, we should consider that audio level is not disturbing them because they tend to be visual learners and use audio only to communicate with other people. According to the usability testing methods developed in the literature, observation techniques are more effective than surveys with autistic populations.

\section{Research Methodology}

For our study, we researched a sample of 14 children, 8 autistic and 6 non-autistic children. We asked them to use two iPad applications: "Tap to Talk" and "Touch to Speak." The study was divided into three main parts. The first part was based on the Eye Tracking usability measurement tool; second part was based on observation and the third part was depended on questionnaires. The second part also divided into two sections: the first section depended on manual 
observation when used an eye tracker as measurement tool and second section was automatic observation using Morae usability-testing software. Morae includes many features, we use the screen recording, observation and analysis of data. The numbers of participants in Eye Tracking study were 7 children, 1 autistic child and 6 non-autistic children. This section of study was limited to one autism child due to the special requirements it placed on the children. Generally, autistic children are restless and cannot stay in the same position for an extended period of time. For the aforementioned reason, we made the second section of study (Morae), which was done by 7 autistic children.

The children were requested to use each application for 10-15 minutes and then fill out the questionnaires. Among the 6 non-autistic participants, 3 were in the age range of 3-6 years. Their parents or guardians filled out these children's questionnaires. 3 children were in the range of 7-12 years, and they filled out the questionnaires by themselves. The 8 autistic participants were in the age range of 5- 12 years. Their teacher filled out these children's questionnaires because their literacy skills are limited.

As Nielsen argues [17], the advantage of small sample sizes is simply return on investment: testing costs increase with each additional study participant. Because 5-6 participants will find $80 \%$ of usability problems, there is little additional benefit to running more than 5 to 6 people through the same study.

Our study of children was conducted in the usability lab of the girls' campus of King Saud University and Autism Center, Riyadh, Saudi Arabia. All the participants of the study were assured that their personal information would be kept confidential. The participants were asked to accomplish 5 tasks for each application.

The tasks that we selected for this study is as the following:

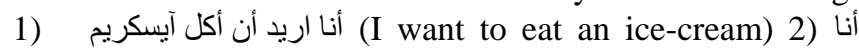
أنا أريد أن ألعب بالكرة (I want to play a ball) 4) العودة للصفحة الرئيسية (5eturn to home page). Choosing these tasks based on varying in the level of difficulty. In addition, these tasks are in Arabic language; however, there is a deferent between two accents, which are the Modern Standard Arabic (MSA), and the classical Arabic. To test the first part, eye tracking was used to produce feedback, to assess how children both with and without ASD react to the two applications. By identifying their areas of interest and their patterns of eye movement, we will be better informed about child preferences. This in turn will contribute to a clearer understanding of children's needs. In the second part, when we used automatic observation, the Airservers were used to mirror the display and action from iPad device into a computer and Morae record it. Fig. 1 presents the procedure that we followed during the case study.

In the study, we focused on the following factors to evaluate the two applications: Ease of use, Learnability, Feedback, Number of errors, Time on task, Satisfaction, Predictability, and Completion time.

Children were the targets of our study. We designed the questioners to attract them, so we used the fancy toolkit scale. For each question in the questionnaire, the participants were to specify the extent of their agreement or disagreement using a five-face scale. The scale ranged from "strongly agree" (happy face) to "strongly disagree" (sad face). As for the remaining three questions, they were there to get the participants' overall perception of the application. They asked the user about the positive, negative and must-have features of the applications.

The decision to perform usability testing on those particular two applications was based on the demand for AAC applications. Several studies observed that AAC applications promote the independence of students with ASD, improve their engagement, and reduce teaching time, therefore enhancing and easing the education process for them [18]. This study aims to extend the therapy beyond clinical settings and to facilitate communication in Arabic-speaking contexts [19]. By identifying usability problems, collecting qualitative and quantitative data and determining the participants' satisfaction with the product, the problems will be identified and the developers will improve the design in subsequent versions. In addition, identifying problems that children was faced will encourage developers to avoid making the same mistakes in developing future AAC applications.

The applications that we have analyzed for this study are both from the iPad domain. There is only one free AAC Arabic application available for autistic people in the App Store, and its name is "Touch to Speak." The other applications are in English. However, "Tap to Talk" is a free application available in an English-language version and the user can purchase an Arabic-language customization.

Both applications are customizable AAC tools for autism, Down syndrome, apraxia, cerebral palsy, aphasia, and any non-verbal condition. Also, they make communication fun, like another "game" on this device. Tap a picture and the application speaks. Each picture can lead to another screen of pictures. The main purpose of both the apps is to assist autistic people in communicating.

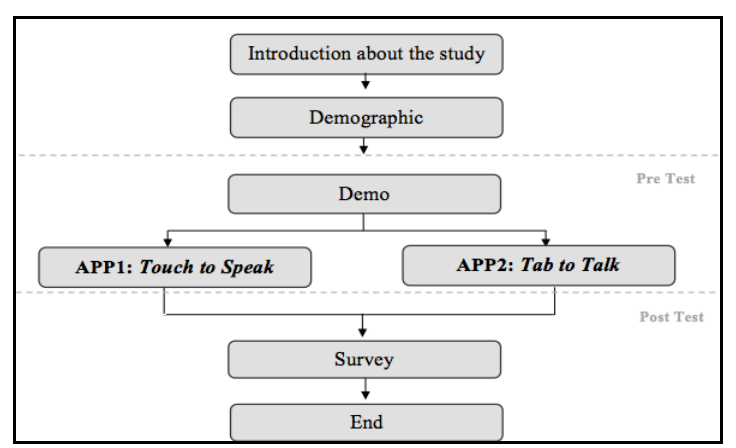

Fig. 1. Work flow procedure for measuring the applications.

The first application under analysis is:

\section{A. Tap to Talk [20]}

This application supports multiple languages. It is compatible with different operating systems: you can play your Tap to Talk albums on iPad, iPhone, iPod touch, Kindle Fire, Nook Color, Nook Tablet, Android devices, Nabi Tablet, Windows 8/RT devices, PC or Mac. On other hand, you can customize your albums or sounds with Tap to Talk Designer on any PC, Mac or iPad. It is basically a mode of communication that formulates grammatically correct sentences using a list of pictures and reads them 
(text-to-speech), as displayed in 0 .

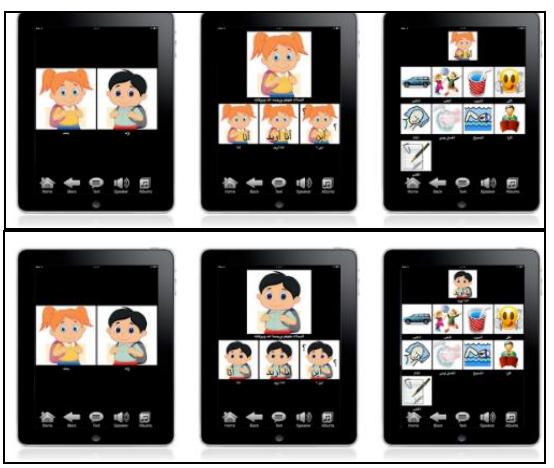

Fig. 2. Application 1: Tap to talk.

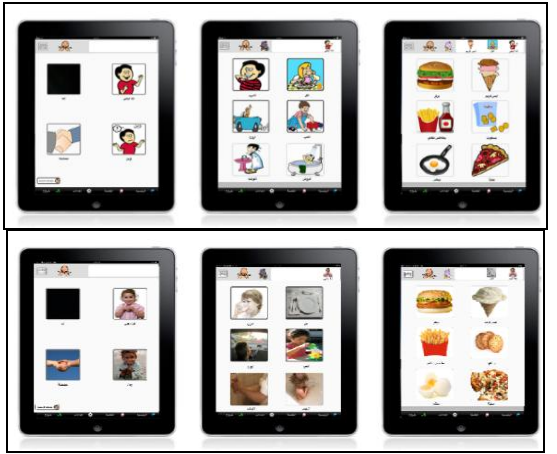

Fig. 3. Application 2: Touch to speak.

\section{B. Touch to Speak [21]}

This application supports only the Arabic language and emphasizes supporting verbal communication in local dialects as well as Modern Standard Arabic (MSA). The application focuses on MSA because of the dominance of using local dialects in clinical settings for speech and language therapy as well as everyday communication in non-formal settings such as at home, leisure, and in most contexts in schools and at work [20]. The items in the application are divided into four main categories, namely, I, I want, where and conversation. The user can customize his/her profile by selecting clipart emoticons or personal photographs. Moreover, users can replace and customize any picture in this application. Under each picture, there is a text that helps the user as shown in Fig. 3.

\section{COMPARATIVE ANALYSIS AND RESUltS}

\section{A. Based on Usability Measurement of Eye Tracker}

Our goal for this usability study was to see if autistic children could focus on the target picture with minimum vacillation between the target picture and other similar ones and easily formulate any sentence. In our first step to perform this study, we elected to use eye tracker, which is the best method to measure the fixation of attention and to measure if we achieved our goals or not. Because we only have a Tobii $\mathrm{x} 120$, we faced many challenge regards setting up the Tobii $\mathrm{x} 120$ on mobile devices (iPads). We used the basic monitoring setup and changed the configuration setup according to the iPad screen size. 0 presents our setup for the usability testing, which was performed in the usability lab of King Saud University.

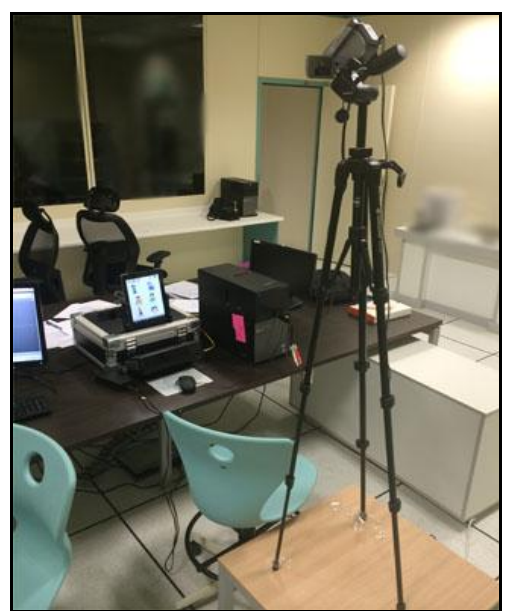

Fig. 4. Basic monitor setup using Tobii x120 and iPad device.

We conducted our usability study using eye tracker on non-autistic children with varying ages and one autistic child. 0 illustrates the heat map of the two applications when using pictures to formulate this sentence "where is my dad." Generally, all participants performed the task correctly in both applications. However, when we asked the participants to formulate another sentence, "I am sad" as shown in 0 , results varied. In Tap to Talk, the participants were confused by the variety of pictures representing sadness; older children who could read the text under each pictures selected the right picture much more quickly and easily than younger ones who had to choose based on the pictures alone. In Touch to Speak, the participants had to move to the second page to locate the target pictures, so some may not have realized that there were other pages. This may also have allowed younger children to fall into choosing similar, but incorrect, pictures.

The autistic child belonged to the category of older children and was familiar with both applications. He could perform the task as easily as a non-autistic child.

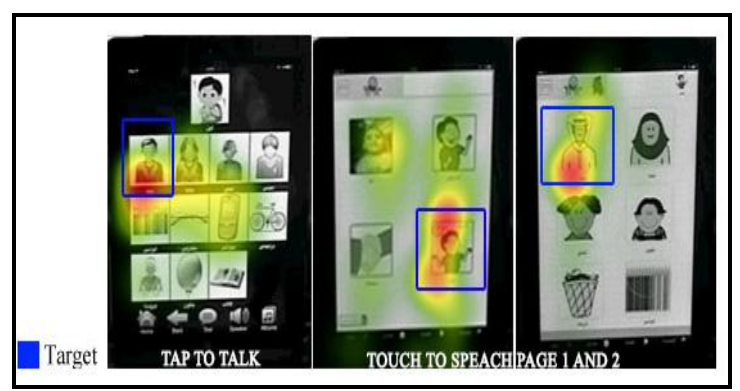

Fig. 5. Heat map of sentence "Where is my dad?".

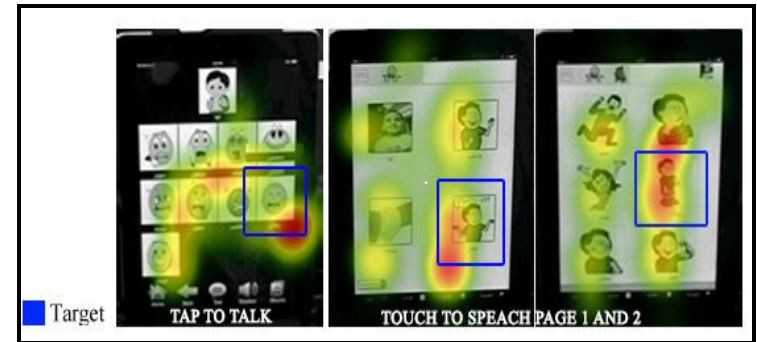

Fig. 6. Heat map of sentence "I am sad".

The following charts (0 and 0$)$ summarize the result of "fixation before," which is defined as the number of times the participants fixates on the media before fixating on an Area of Interest (AOI) or AOI group for the first time. It is used to measure the cognitive efforts to reach the target, an AOI, 
when formulating this sentence "I am sad". From below figures we conclude that, younger children have a higher percentage of fixation before reaching the target, and that was because they had to formulate the sentence using pictures only. Younger children are similar to autistic children in this case because autistic people are visual learners and all have to rely on pictures to formulate any sentence.

In addition, the availability of other pages is unpredictable to younger children. Therefore, they wasted too much time in fixation on the first page of the application "Touch to Speak," although the target was on the second page.

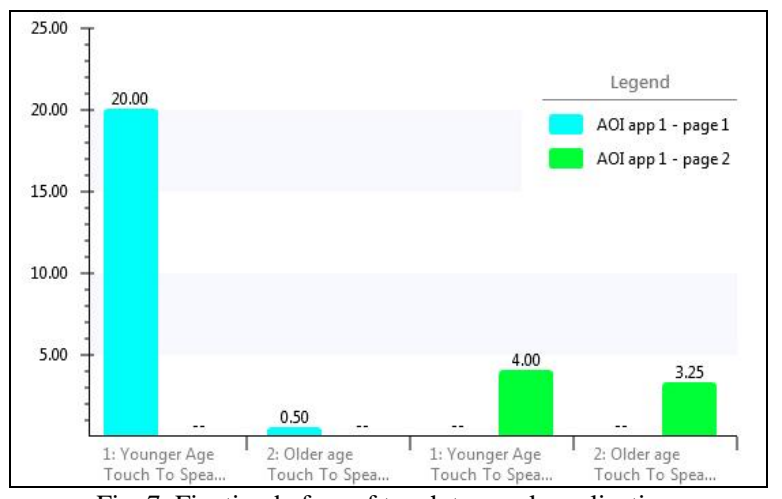

Fig. 7. Fixation before of touch to speak application.

\section{B. Based on Observer Opinion}

\section{1) Manual observation}

While conducting our study on participants using eye trackers, we observed the children at the same time to analyze their effectiveness at performing each task based on four characteristics: Learnability, Time on task, Completion time, and Number of errors per task.

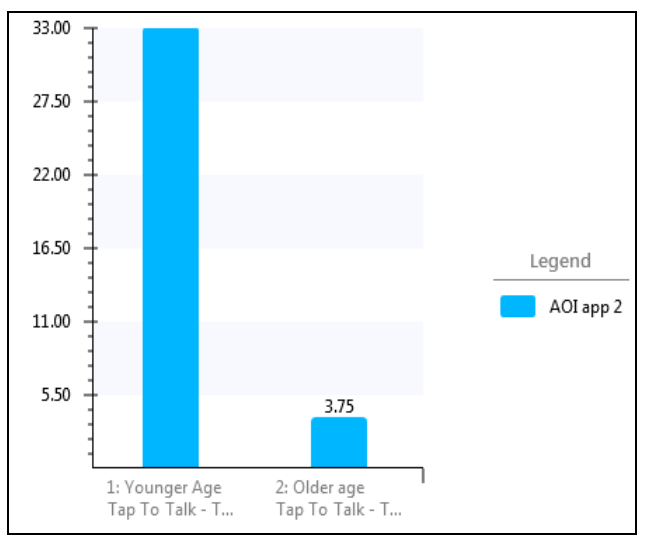

Fig. 8. Fixation before of tap to talk application.

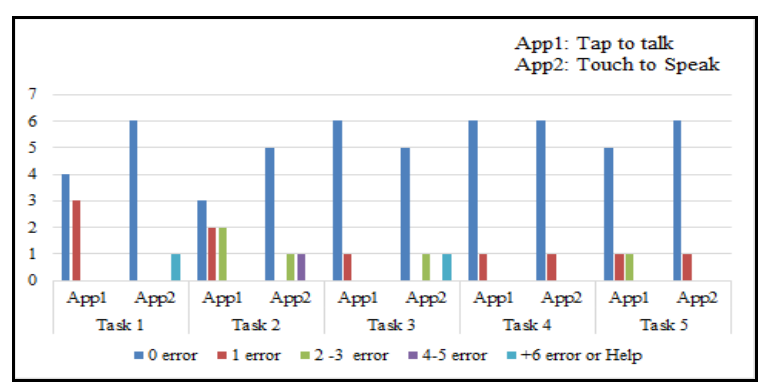

Fig. 9. Number of errors in each task of App1 and App2.

We first described the completion time, which is the time it takes to finish the five tasks for every application for all participants. Learnability affected completion time. To avoid learnability issues and how they might bias our results, we changed the order in which the participants tested the applications. In most cases, participants performing the second application completed it more quickly and easily than the first one.

In Fig. 9, we describe the effectiveness of performing each task based on the number of errors. Learnability also reduces the number of errors. We found that the number of errors was higher in "Touch to Speak," and we think that this happened because the younger children were distracted by the higher numbers or unaware that there were images on other pages. We ordered the participants in the figures based on their ages: $\mathrm{P} 1$ is older than $\mathrm{P} 2$ and so on.

\section{2) Observation using Morae}

After the test of the two applications was completed, we analyzed the data for all participants in accomplishing each task. The following graphs describe the error rate and average time in accomplishing each task.

The number of errors in the Tap to Talk application was lower in most tasks. However, in task 1, Touch to Speak performed better, while task five have the same value.

Based on average time to accomplish each task, Task one and four required less time to accomplish in Tap to Talk. However, task two, three and five took less time in the second application, Touch to Speak.

\section{Based on a Questionnaires}

We conducted our study on two cases: in the first case, non-autistic participants using eye tracker, and in the second case, autistic pediatricians using Air Server and Morae ${ }^{\circledR}$. We asked participants' guardians to fill out the questionnaire in case of younger and autistic children. While an older children filled the questionnaire by themselves. Our questions were based on four characteristics:

- Ease of use: rated each application based on the simplicity to perform the tasks.

- Feedback: rated participants' or their guardians' perspectives on each application.

- Satisfaction: rated how they felt about each application.

- Predictability: rated the ease of interpreting the images without reading the text under each one and formulating sentences using pictures only.

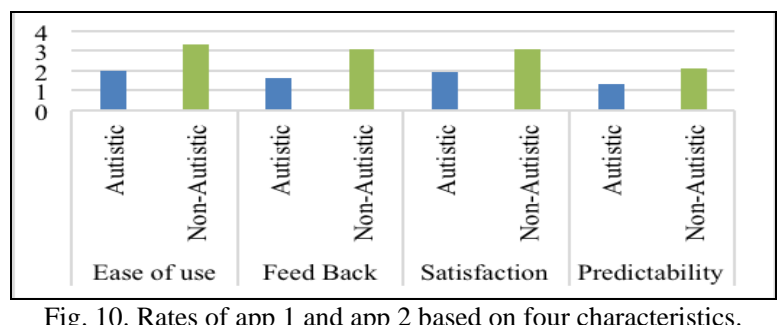

In both cases, we ordered the participants according to their ages. In the figure below, the number 5 means is completely satisfied, 4 is often satisfied, 3 is little satisfied, 2 is not satisfied and 1 is frustrating. 0 illustrates how each non-autistic participant's guardians and autistic participants' teachers rated each application according to the specified characteristics.

We handed a questionnaire to participants, their guardians 
or teachers after each session to rate both applications according to the usability measurement. We also asked them to suggest new features to improve each application. They Stated the main advantage of Tap-to-Talk application as "displaying large number of pictures in one page facilitate the selection for children" while in Touch-to-Speak "MSA language is used, so it is easier as to used as informal communication in children daily lives." Most agreed that both applications are easy-to-use for non-speaking children to use in their daily lives and express their needs and one of the means of alternative communication.

They also stated the main disadvantages for Tap-to-Talk, as "center picture inside each category is distracting the users "while Touch-to-Speak" too many pages and smaller number of pictures on each page. Most confess that in both applications child must always have iPad with him/her. Furthermore, using the iPad application for communication makes autistic children dependent on it rather than attempting to speak

They suggest new features in Touch-to-Speak is to reduce the number of pages in each category or clarify the presence of other pages

When we made a test in "Tap to Talk". We used built-in text-to-speech feature that provided from the application. Unfortunately, many participants wrote that sounds of words are unclear. However, application has the ability to customize the sounds of any pictures. Therefore, it is not an issue.

\section{DISCUSSION}

The main challenge in working with autistic children is that they prefer to deal with people they know. In addition, some of children were using the iPad for the first time.

Using these applications is generally straightforward, although some pictures are unclear. Both applications have the ability to customize any pictures to suit the user's needs. However, just as both applications have a set of advantages, two main issues arose: in the application "Tap to Talk," the picture at the top of each page is distracting. The other issue that emerged with "Touch to Speak" was the unpredictability of the presence of other pages.

From our study, we will propose a recommendation for an optimal design of ideal AAC application. This design will combine advantages for both applications in one and avoid the issues that appeared in them. We will suggest some new features from our experience that we think it is helpful to include in the recommended application. These suggestions based on our observation for who participate in this study. Our design is very similar to "Touch to Speak" with some improvements. The main different are:

- The number of pictures that appear in each page should be within the acceptable limit of pictures. We think 16 are enough to have clear picture according to the size of iPad.

- The home page icon should be distinguished. Also there should be an icon to clarify the apparent of other pages like "Next Page" icon, which is appear when there is multiple number of pages.

- There should be a tutorial to guide the users about the functionality of the application.

- There might be an ability to hide or unhide the text.
- There should be an ability to pronounce the completely formulated sentence.

The similarities are customize voice and pictures, used MSA language, categorization, password protection for setting, can use keyboard to write and pronounce the text using build in voice, and can add any formulated sentence to favorite.

\section{FUTURE WORK}

Autistic children alike non-autistic children at young ages (3 to 6) would quickly get bored with sitting still during calibration and while performing all tasks. In addition, using Tobii X120 for them was inefficient due to the difficulty of maintaining them in a fixed position during the whole process. In our future study, we aim to use eye tracker glasses, also called mobile eye trackers, on non-autistic children and try it on children with ASD to make them more comfortable and get more accurate results. Tobii glasses are more efficient for uncontrolled situations.

\section{CONCLUSION}

Autism spectrum disorder (ASD) cases are increasing daily. In 2002, there were 42,500 confirmed cases of autism in Saudi Arabia (with a population under 23 million), and many more cases are undiagnosed [22]. This paper has analyzed two existing iPad applications to facilitate communication and discussed design mistakes that are dissatisfying to autistic children. Our result based on our manual observation and automatic observation using Morae, also result from using eye tracker as usability tool. At the end we proposed some suggestions for AAC applications that designed for autistic children.

\section{ACKNOWLEDGMENT}

We appreciate the support of King Saud University throughout this research. Also, we would like to thank Dr. Areej Al-Wabil and Dr. Khalid Al-Nafjan for their help and advice. We extend our appreciation to the Deanship of Scientific Research at King Saud University for funding the work through the research group project number RGP-VPP-157. Finally, we would like to thank all the children who participated in our research and the Mother of the Prince Faisal bin Fahd Center for Autism that helped us to perform our usability test study in their center.

\section{REFERENCES}

[1] C. Nordqvist. (June 27, 2014). What Is Schizophrenia? Medical News Today. [Online]. Available: http://www.medicalnewstoday.com/articles/36942.php.

[2] G. R. Hayes, S. Hirano, G. Marcu, M. Monibi, D. H. Nguyen, and M. Yeganyan, "Interactive visual supports for children with autism," Pers Ubiquit Comput, vol. 14, no. 7, pp. 663-680, Oct. 2010.

[3] Diagnostic and Statistical Manual of Mental Disorders, 4th ed., Washington, DC: American Psychiatric Association, 2000.

[4] Autism Society of America. (June 27, 2014). About Autism. [Online]. Available: http://www.autism-society.org/about-autism/

[5] Centers for Disease Control and Prevention. (June 27, 2014). Facts about ASDs. Autism Spectrum Disorder (ASD). [Online]. Available: http://www.cdc.gov/ncbddd/autism/facts.html 
[6] A. Alliano, K. Herriger, A. Koutsoftas, and T. Bartolotta, "A review of 21 iPad applications for augmentative and alternative communication purposes," Perspectives on Augmentative \& Alternative Communication, vol. 21, no. 2, p. 60, June 2012.

[7] Wikipedia, "Mobile app," June 27, 2014.

[8] T. Treeratanapon, "Design of the usability measurement framework for mobile applications," presented at International Conference on Computer and Information Technology (ICCIT'2012), Bangkok, 2012.

[9] Guidance on Usability, ISO Standard 9241- 11, 1998.

[10] American Speech-Language-Hearing Association (ASHA). (June 27, 2014). Augmentative and Alternative Communication (AAC). [Online]. Available: http://www.asha.org/public/speech/disorders/AAC/

[11] C. Yamagata, M. Kowtko, J. F. Coppola, and S. Joyce, "Mobile app development and usability research to help dementia and Alzheimer patients," in Proc. Systems, Applications and Technology Conference (LISAT), 2013, pp. 1-6.

[12] L. Burkhart. (June 27, 2014). ISAAC - What is AAC? [Online]. Available: http://www.isaac-online.org/english/what-is-aac/

[13] M. Barry, A. Kehoe, and I. Pitt, "Usability Evaluation of Educational Game Software for Children with Autism," in Proc. World Conference on Educational Multimedia, Hypermedia and Telecommunications, Vienne, 2008, pp. 1366-1370.

[14] F. Din and J. Calao, "The effects of playing educational video games on kindergarten achievement," Child Study Journal, vol. 31, no. 2, pp 95-102, 2001.

[15] C. Clanton, "An interpreted demonstration of computer game design," in Proc. CHI 98 Conference Summary on Human Factors in Computing Systems, New York, NY, USA, 1998, pp. 1-2.

[16] S. Khan, M. N. Tahir, and A. Raza, "Usability issues for smartphone users with special needs - Autism," in Proc. International Conference on Open Source Systems and Technologies (ICOSST), 2013, pp. $107-113$.

[17] J. Nielsen, Usability Engineering, 1 ed., San Francisco, Calif.: Morgan Kaufmann, 1993.

[18] M. Cramer, S. H. Hirano, M. Tentori, M. T. Yeganyan, and G. R. Hayes, "Classroom-based assistive technology: Collective use of interactive visual schedules by students with Autism," in Proc. the SIGCHI Conference on Human Factors in Computing Systems, New York, NY, USA, 2011, pp. 1-10.

[19] B. Al-Arifi, A. Al-Rubaian, G. Al-Ofisan, N. Al-Romi, and A. Al-Wabil, "Towards an Arabic language augmentative and alternative communication application for Autism," in Design, User Experience, and Usability. Health, Learning, Playing, Cultural, and Cross-Cultural User Experience, A. Marcus, Ed., Berlin Heidelberg: Springer, 2013, pp. 333-341.
[20] TouchToSpeak. (June 27, 2014). App Store. [Online]. Available: http://itunes.apple.com/us/app/swty/id689685810?mt=8

[21] TapToTalkTM. (June 27, 2014). App Store. [Online]. Available: https://itunes.apple.com/us/app/taptotalk/id367083194?mt=8

[22] F. Yazbak. (2003). Regressive Autism and MMR Vaccination. [Online]. Available: http://www.whale.to/vaccine/yazbak1.html

Lyan Al-Wakeel received her bachelor degree in information technology from King Saud University in 2011. She is a master student in software engineering, College of Computer and Information Sciences at King Saud University, Riyadh, Saudi Arabia.She works as a teaching assistant in Dept of Computer Science, College of Computers and Information Technology at the University of Tabuk, Tabuk, Saudi Arabia. Ms. AlWakeel is a member in Software Knowledge Engineering Research Group (SKERG). Her research is related to human-computer interaction.

Amjad Al-Ghnaim received the bachelor degree in information technology from King Saud University (KSU) in 2010. Her graduation project is focused on image and video processing and it had been done with group of six students. Furthermore, they had a one published paper, which is "I See What You Say (ISWYS): Arabic lip reading system, Dubai, UAE, IEEE, 2013”. AlGhanim is a master student at Software Engineering Department, KSU, Riyadh, Saudi Arabia. She is a freshly researcher and her researches interests include assistive technology, usability engineering and requirement engineering. Nowadays, she focuses on privacy engineering.

Shaden Al-Zeer is a MSc student in Software Engineering Department of at King Saud University; she received her BSc. degree in information technology from King Saud University (KSU) in 2010. She worked as a teaching assistant in Computer Sciences Department, Hurrymila college of Science and Humanities, Shaqra University. Ms. AlZeer is a member in Software Knowledge Engineering Research Group (SKERG). She has with her graduation project team a Best Student Paper Awards in Current Trends in Information Technology (CTIT), Dubai, UAE, IEEE, 2013 International Conference, which is "I See What You Say (ISWYS): Arabic lip reading system". Her research areas include assistive technology and human-computer interaction.

Khalid Al-Nafjan is an associate professor in Software Engineering Department, College of Computer Science, at King Saud University in Riyadh, Saudi Arabia. He has obtained his bachelor degree from Information Systems Department at that university, and $\mathrm{MSc}$ and $\mathrm{PhD}$ in software engineering from Computer Science Department in Sheffield University, UK. Dr. AlNafjan research interests lie in software engineering education, Systems of systems engineering and $\mathrm{C} 4 \mathrm{i}$, and software usability. 